
\title{
3 Research Soure \\ Human Recombinant Fibroblast Growth Factor-18 (Sprifermin), Enhances Cartilage Healing in a Cartilage Injury Model
}

\section{Honey Hendesi}

University of Pennsylvania Perelman School of Medicine

\section{Suzanne Stewart}

University of Pennsylvania School of Veterinary Medicine

Michelle L Gibison

University of Pennsylvania School of Veterinary Medicine

\section{Hans Guehring}

Merck KGaA

\section{Dean W. Richardson}

University of Pennsylvania School of Veterinary Medicine

George R Dodge ( $\sim$ gdodge@pennmedicine.upenn.edu )

\section{Research article}

Keywords: FGF-18, sprifermin, cartilage repair, osteoarthritis, microfracture, equine animal model

Posted Date: May 23rd, 2020

DOI: https://doi.org/10.21203/rs.3.rs-29880/v1

License: (a) (i) This work is licensed under a Creative Commons Attribution 4.0 International License. Read Full License 


\section{Abstract}

Background: Post traumatic osteoarthritis is a disabling condition impacting mostly young and active population. In the present study we investigated the impact of intra-articular sprifermin, a recombinant truncated fibroblast growth factor 18 , on the outcome of microfracture treatment, a widely used surgical technique to enhance cartilage healing at the site of injury.

Methods: For the purpose if this study, we created a cartilage defect and performed microfracture treatment in fetlock joints of 18 horses, treated joints with one of three doses of sprifermin $(10,30$, or $100 \mathrm{mg})$ or with saline, hyaluronan ( $\mathrm{HA})$, and evaluated animals functional and structural outcomes over 24 weeks. For primary outcome measures we performed histological evaluations and performed gene expression analysis of aggrecan, collagen types I and II, and cartilage oligomeric matrix protein (COMP) in three regions of interest. As secondary outcome measures we examined animal's lameness, performed arthroscopic, radiographic, and CT scan imaging, and gross morphology assessment.

Results: We detected the highest treatment benefit following $100 \mathrm{mg}$ sprifermin treatment. Overall histological assessment showed an improvement in the kissing region and expression of constitutive genes showed a concentration dependent enhancement, especially in the peri-lesion area. We detected a significant improvement in lameness scores, arthroscopic evaluations, radiography, and CT scans following sprifermin treatment when results from three dose-treatment groups were combined.

Conclusion: Our results demonstrated for the first time, an enhancement in outcomes of the microfracture treatment following sprifermin treatment, suggesting a cartilage regenerative role and a potential benefit of sprifermin treatment in early cartilage injuries.

\section{Background}

The poor healing capacity of articular cartilage is a well-known challenge in managing cartilage injuries and is a contributing factor to arthritis and especially to post-traumatic osteoarthritis (PTOA) [1, 2]. According to the Center for Disease Control and Prevention, one in four U.S. adults suffers from arthritis and the total annual direct medical cost of arthritis exceeds 81 billion dollars [3]. Osteoarthritis is the most common type of arthritis and it is the third most rapidly growing cause of disability behind diabetes and dementia. In addition, osteoarthritis is the most prevalent co-morbid disease associated with a $55 \%$ increase in mortality rate of patients [4]. The catastrophic individual and societal burden of osteoarthritis underscores the importance and urgency of developing reliable interventions to decrease the incidence and restrain the progression of osteoarthritis.

To improve cartilage healing following injuries, a variety of surgical techniques have been developed and applied by surgeons, with microfracture treatment being the most practical and widely performed $[5,6]$. In this technique, small perforations are made into the subchondral bone to allow bleeding, and as a result marrow derived mesenchymal cells and cartilage progenitors can access the defect site [7-9]. Although the presence of progenitor cells improves filling of the defect, studies have shown that the quality of newly formed cartilaginous tissue is often more fibrous than hyaline $[7,10,11]$. Hyaline cartilage is the optimal form of articular cartilage and is predominantly made of collagen type II, has high water content, remarkably low friction and high load- 
bearing capacity [12]. In contrast, fibrocartilage, which is naturally present in meniscus, is rich in collagen type I and compared to hyaline cartilage, is less shock-absorbing, inferior weight-bearing ability and is biomechanically inferior $[12,13]$. The quality of cartilage is a critical factor in the durability and functionality of repair tissue, and thus an important consideration in the long-term outcome of microfracture surgery [8]. Accordingly, surgeons apply various growth factors (e.g. BMP7, BMP2, TGF and PRP) in combination with microfracture treatment to support formation of hyaline repair cartilage [14-17]. In spite of all advancements in surgical techniques, improving the long term outcome of the microfracture treatment remains a challenge [8]. Solheim and colleagues reported poor outcome in $46 \%$ of patients $10-14$ years post-surgery with $39 \%$ of cohorts required additional surgical procedures following the original microfracture surgery [18].

One of the growth factors with a well-known anabolic effect on cartilage is fibroblast growth factor-18 (FGF18) [19-23]. FGF18 is a member of the FGF family of growth factors which play a central role in skeletal growth and development [24-26]. A study on FGF18 null mice detected numerous bone and joint abnormalities including a delay in calvarial suture closure [19]. Previous studies have supported a role for FGF18 in chondrocytes proliferation and differentiation as well as cartilage matrix hemostasis. These studies demonstrated that FGF18 increases collagen type II, glycosaminoglycan and chondroitin sulfate expression and reduces collagen type II breakdown following cartilage injury [21, 22, 26, 27]. Additionally, intra-articular injection of FGF18 in a rat osteoarthritis model reduced cartilage degeneration, induced new cartilage formation and indicated a potent in vivo anabolic effect for FGF18 [28]. This potential capability of FGF18 and the necessity for new and effective Disease Modifying Osteoarthritis Drugs (DMOADs) inspired development of sprifermin, a truncated recombinant human FGF18 (Merck KGaA, Darmstadt, Germany). In vitro experiments utilizing sprifermin have demonstrated an improvement in collagen type II:I ratio and hyaline cartilage matrix formation and revealed a "hit and run" mode of action for sprifermin [29]. In an ex vivo study, Sennet and colleagues showed sprifermin can increase collagen content and adhesive strength leading to better cartilage-cartilage integration and superior repair of a microfracture defect [30]. Sprifermin is currently in phase two of clinical trials for treatment of knee osteoarthritis. A 12-months clinical study on168 patients showed that sprifermin treatment was associated with statistically significant, dose-dependent improvement of lateral femorotibial cartilage thickness. In addition, this study demonstrated a significant decrease in pain scores in patients who received sprifermin [31]. In the report of a recent 2-years clinical study, MRI results showed significant dose dependent modification of cartilage thickness in the total femorotibial joint (TFTJ), medial and lateral femorotibial compartments (MFTC, LFTC), and central medial (cMFTC) and central lateral (cLFTC) TFTJ sub-regions [32]. The above mentioned clinical studies and a study by Dahlberg et al. on 55 patients scheduled for total knee replacement, have shown efficacy and safety of intra-articular sprifermin in patients with symptomatic radiographic knee osteoarthritis detected no local or systemic safety concerns associated with sprifermin injections [33]. Two animal studies on an ovine model supported the beneficial effect of intra-articular sprifermin treatment on cartilage repair: Power and colleagues showed an improvement in cartilage defect fill and collagen type II expression in sprifermin treated ovine joints and Howard et al., proved an enhancement in weight bearing, histology scores and collagen type II expression following sprifermin treatment [34].

The result of a pre-clinical animal study can best be extrapolated to a human condition in an appropriate animal model that more closely represents the human condition. Horses are considered as an excellent animal model for preclinical evaluation of new cartilage repair techniques and technologies $[35,36]$. 
In the present study, we have used an equine animal model to investigate the effect of a three dose intraarticular injection treatment regimen of sprifermin on the outcome of microfracture surgery in a cartilage defect model (Fig. 1). Treatment with sprifermin was well tolerated and safe and by performing ante-mortem and postmortem evaluations, we detected a significant improvement in functional and structural aspects of the cartilage repair tissue as a result of sprifermin treatment.

\section{Methods}

\section{Experimental Design}

Eighteen healthy horses (age range 2-7 years) were enrolled in the experimental study approved by the University of Pennsylvania Animal Care and Use Committee. All horses underwent physical and lameness examinations, including radiographic evaluation of all four fetlock (metacarpophalangeal and metatarsophalangeal) joints prior to study inclusion. Only horses free of lameness and fetlock disease were included in the study.

On day 0 , each horse was placed under general anesthesia in right lateral recumbency for arthroscopy of all four fetlock joints. Arthroscopic portals were created in the palmar aspect of either the right or left metacarpophalangeal joint and a $15 \mathrm{~mm}$ circumferential full thickness articular cartilage defect was created using a combination of an arthroscopic burr and currettes, on the central dorsal aspect of the lateral proximal sesamoid bone. The defect extended approximately $2 \mathrm{~mm}$ from the most distal aspect of the bone to approximately $2 \mathrm{~mm}$ from the abaxial border of the bone to the size of $15 \mathrm{~mm}$. Discs made of radiographic film were used as templates to ensure the defects were of uniform size (Fig. 1A). A 45 or a 90-degree calibrated awl was used to micropick 25-30 locations within the defect. Arthroscopy portals were made as previously described in the contralateral metacarpophalangeal joint and the left plantar medial and right plantar lateral metatarsophalangeal joints. A cartilage defect was created in the lateral proximal sesamoid bones and micropicked as previously described in 3 out of four fetlock joints. One fetlock joint had a sham procedure where no defect was created. This served as the control limb. The arthroscopic and instrument portals were closed using a simple interrupted pattern with 2-0 polypropylene with light adhesive bandages placed for recovery. All study horses received a single intraoperative dose of phenylbutazone at $4.4 \mathrm{mg} / \mathrm{kg}$ intravenously.

Bandages were changed every 3-5 days and maintained until suture removal at 10 days post operatively.

Animals were placed in their assigned stall $(3.65 \times 3.65 \mathrm{M})$ for the two initial weeks post operatively and transitioned to free paddock turnout from weeks 3-12 post operatively. Sutures were removed at 10 days postoperative using aseptic technique and light bandages were maintained until the time of suture removal. A second look arthroscopy and biopsy was performed on week 12 and animals resumed turnout from weeks 1424. All animals were humanely euthanized at 24 weeks with an overdose of sodium pentobarbital. All four limbs were harvested for postmortem analysis.

\section{Treatment Groups}


Weekly consecutive intra-articular medication of the testing article was performed from weeks 3-5. One joint each was medicated with 20 mg hyaluronan (Hylartin $\mathrm{V}^{\circledR}$, Pfizer, New York, NY), 2 ml of 0.9\% sterile sodium chloride (placebo) or one of three different doses of sprifermin. Three doses of sprifermin $(10 \mathrm{~g}, 30 \mathrm{~g}$ and $100 \mathrm{~g})$ were tested and six horses were used for each dose for a total of 18 horses (Fig. 1B). Treatment regime was based on the intended route of administration of the test article in humans. Different dose ranges were to be evaluated to determine optimum cartilage healing. Horses were randomly assigned to treatment groups.

\section{Methods: Primary Endpoints}

\section{Histology}

Cartilage samples were harvested from control, lesion and kissing region of fetlocks. Samples decalcified, dehydrated and paraffin embedded before being cut into $7 \mathrm{~mm}$ sections and stained using Safranin 0/Fast green. The slides were scanned on the Aperio ImageScope (Leica Biosystems, Wetzlar, Germany) at 20 x and 5 $\mathrm{mm}$ wide regions of interest were cropped for scoring. Semi-quantitative scoring was conducted by six independent observers blinded to treatment protocol using ten different metrics: (1) matrix staining, (2) cellularity, (3) superficial zone clustering, (4) middle-deep zone clustering, (5) surface architecture, (6) subchondral bone involvement, (7) lesion intensity, (8) superficial zone assessment, (9) middle-deep zone assessment, and (10) overall assessment. To evaluate cell proliferation, number of chondrocytes were counted in each area using 3 regions of interest and manual counting.

\section{RNA Isolation and Real-Time PCR}

Cartilage samples were harvested from lesion, peri-lesion, kissing region and control and immediately frozen in the liquid nitrogen. To isolate the RNA, frozen cartilages were homogenized using a TissueLyser LT (Qiagen, Germantown, MD). Trizol was added to the grounded cartilage samples and Qiagen miRNeasy micro kit was used for RNA extraction. A NanoDrop (Thermo Fisher Scientific, Waltham, Mass) was used to measure the quantity of the isolated RNA prior to synthesizing cDNA using a iScript ${ }^{\mathrm{TM}}$ reverse transcription supermix (Bio-Rad, Hercules, CA) and a Bio-Rad thermo-cycler. Real-time PCR was performed using a Fluidigm Biomark ${ }^{\mathrm{TM}}$ HD platform (Fluidigm, San Francisco, CA) and TaqMan ${ }^{\circledR}$ assays (Applied Biosystems, Thermo Fisher Scientific) as probes. Prior to the Biomark ${ }^{\mathrm{TM}}$ assay, cDNA samples were pre-amplified for 12 cycles using a mix of Fluidigm PreAmp master-mix and pool of TaqMan ${ }^{\circledR}$ assays. Geometric mean of CT values from five different housekeeping genes (GAPDH, GUSB, ACTB, B2M and SDHA) was used to measure the relative expression of genes of interest.

\section{Methods: Secondary Endpoints}

\section{Clinical Assessment}


Horses were assessed daily by animal care personnel and lameness examinations performed at weeks $3,4,5$, $10,12,14,18,22$ and 24 by a single blinded observer board certified in large animal surgery. Lameness was graded on a standardized scale of 0-5 according to American Association of Equine Practitioners guideline on a straight line and a circle. Fetlock flexion tests were graded separately on a scale of 0-3 [37].

\section{Synovial Fluid Analysis}

Synovial fluid was obtained from all four fetlock joints at weeks $0,3,4,5,10,12,14,18,22$ and 24. Color, clarity, total protein (TP) concentration, and white blood cell (WBC) count were determined for synovial fluid via routine methods.

\section{Second Look Arthroscopy}

Arthroscopic evaluation of the cartilage defect was performed at 12 weeks. Videos obtained at the time of surgery were reviewed and the gross morphology of the cartilage defect scored by two blinded observers. The color of the repair tissue, the surface integrity of the repair and the degree of coverage was evaluated on a scale of 1-5 (1 represented severe change and 5 was normal; Table S1). The scoring method applied was adopted from Van den Borne et al, 2007 and Goebel et al, 2012 [38, 39].

\section{Gross Joint Evaluation}

For each limb, the surgical site was inspected for evidence of inflammation, soft tissue healing, discharge, and soft or hard tissue swelling. Subcutaneous tissues were incised and evaluated for evidence of inflammation or fibrosis. The fetlock joints were disarticulated and the synovial membrane, joint capsule, and all articulating surfaces within the operated joint were examined and macroscopically evaluated and scored [38-40]. Digital photographs were obtained of the cartilage defect (where applicable), and of the articular cartilage on the lateral condyle and proximal palmar or plantar articulating surface of the proximal phalanx (P1). Digital photographs were scored by two blinded observers using the follow scoring system (Table S2).

\section{Radiographs}

Survey radiographs (Lateral-medial, DMPLO, DLPMO and Dorsopalmar/plantar) of all four fetlocks were obtained for all animals following enrollment in the study, at 12 weeks post operatively and at termination (week 24). The radiographs for each individual horse at each time point were scored by two blinded observers. Radiographic images were evaluated for periarticular soft tissue swelling, soft tissue mineralization and development of enthesophytes and osteophytes on a scale of 1-5 (1 represented severe change and 5 was normal; Table S3). The radiographic scoring system was adapted from Boyce et al, 2013 [41].

\section{Computed Tomography}


Computed tomographic (CT) evaluation was performed with a portable 8-slice CT scanner (CereTom ${ }^{\text {TM }}$ OTOscan, NeuroLogica, Danvers, MA) on all four fetlocks of all horses. Initial images were obtained at $2.5 \mathrm{~mm}$ slice thickness and reconstructed to $1.25 \mathrm{~mm}$. Images were viewed on Osirix imaging software and graded by two blinded observers on a scale of $1-5$, with a score of 1 being most severe (Table S4) [42].

\section{Statistical Analysis}

In the case of gene expression and histology data, non-parametric Mann-Whitney test was applied to determine the statistical significant differences in gene expression between groups. Study is not powered for statistical analysis of the secondary end-points. Therefore, the sprifermin groups were combined to assess a putative overall effect. In the case of data from second look arthroscopy, radiography, CT and gross evaluation, a nonparametric Kruskal-Wallis with Dunn's multiple comparison test is used to determine statistical significance within and between groups. For percentage benefits, scores from placebo, HA, and sprifermin treatment groups were compared to those from sham ( $100 \%$ benefit) and no statistical analysis was performed for this underpower measures. $P<0.05$ was accepted as significant in all analysis. No data imputation or baseline adjustments were implemented. GraphPad Prism 8 statistical software (GraphPad Software, Inc, La Jolla, CA) was used for data analysis.

\section{Results}

\section{Results: Primary Endpoints}

As primary endpoints we choose the structural elements of histology and gene expression of constitutive cartilage extracellular matrix and those genes that relate to catabolic activity.

\section{Histological Evaluation}

Histological evaluation of samples from three regions of interest (lesion, kissing region, opposite facing cartilage to lesion, and control; Fig. 2A) showed significant improvement in several metrics as a result of sprifermin treatment.

In lesion, all placebo, HA and sprifermin treated groups showed a fibrotic non-cartilaginous repair tissue and their overall assessment was significantly deteriorated (Fig. 2D). We detected most marked changes in the kissing region where both $10 \mathrm{~g}$ and $100 \mathrm{~g}$ sprifermin groups showed an improvement in repair tissue (Fig. S1).

In the kissing region group, $100 \mathrm{~g}$ sprifermin showed significant improvement in surface architecture $(93 \pm 4.33$ versus $71.34 \pm 26.63, p=0.021$; Fig. S1E), decrease in the lesion intensity (91.94 \pm 11.71 versus $73.48 \pm 25.94, p=$ 0.015 ; Fig. S1G) compared to placebo group (scores and the lesion intensity are inversely related), and a nearly to significant improvement in the overall assessment ( $p=0.057$; Fig. 2E). In the same region, $10 \mathrm{~g}$ sprifermin significantly associated with improved surface architecture compared to placebo (sprifermin $93.98 \pm 4.11$ versus placebo $71.34 \pm 26.63, p=0.009$; Fig $S 1 E$ ) and five out of six animals showed improvement in overall assessment when compared to placebo (Fig 2E). For the kissing region group in the $30 \mathrm{~g}$ sprifermin group, there 
was an opposite trend detected ( $\mathrm{p}<0.05)$ with reduced cellularity, increased clustering, and disrupted surface architecture when compared to sham joints (Fig. S1).

In the control region group, except for a significant decrease in the overall assessment (Fig. 2C) and cellularity scores following treatment with $10 \mathrm{~g}$ sprifermin, and a decrease in surface architecture score in $30 \mathrm{~g}$ group, other metrics for $10 \mathrm{~g}$ and $30 \mathrm{~g}$ groups and all metrics for $100 \mathrm{~g}$ group were comparable with sham. In addition, in lesion, kissing region, and control groups, sprifermin treatment resulted in an increase in the average cell number. This increase was significant in the lesion of the $10 \mathrm{~g}$ group; Fig. S1, K to M).

\section{Gene Expression}

We isolated RNA and evaluated gene expression in three regions: lesion, peri-lesion, and kissing region (Fig. 2A). In peri-lesion group receiving $100 \mathrm{~g}$ sprifermin, there was a significant increase in aggrecan $(p<0.05)$ and COMP $(p<0.01)$ expression compared to HA and placebo groups (Fig. $3 A$ and $C$ ). In the same treatment group, the average expression of collagen type II increased (100 g sprifermin $4885 \pm 3053$ versus placebo $3031 \pm 2672$ ) and 5 out of 6 animals showed an improvement in collagen type II expression when compared to the average gene expression in placebo and HA groups (Fig. 3B). In the case of collagen type I expression, we detected a significant decrease compared to $\mathrm{HA}$ and placebo treatment groups (Collagen type I $1.068 \pm 0.57$ versus HA $11.78 \pm 8.29$ and placebo $20.64 \pm 27.63, p=0.0058$ and $p=0.018$ respectively; Fig. 3D).

In kissing region group, we detected a dose dependent increase in average aggrecan, collagen type II and COMP expression with highest expression in $100 \mathrm{~g}$ (Fig. $3 \mathrm{E}$ to G). In $100 \mathrm{~g}$ sprifermin, COMP expression showed a near to significant $(p=0.055)$ and significant $(p=0.018)$ increase in expression compare to placebo and HA treated joints. Although we did not detect any significant changes in collagen type I expression, all animals except one showed a decrease in gene expression compared to the average of the gene expression in placebo and $\mathrm{HA}$ groups (0.0578 and 1.001 respectively; Fig. $3 \mathrm{H}$ ).

In $100 \mu \mathrm{g}$ sprifermin treated lesion group, when compared to placebo, there was an increase in level of aggrecan, collagen type II and COMP expression and a decrease in level of collagen type I expression, however these changes were not significant $(p=0.453,0.922,0.103$, and 0.136 respectively; Fig. $3 \mathrm{I}$ to $\mathrm{L}$ ). Five out of six animals treated with $100 \mu \mathrm{g}$ sprifermin showed an improvement (reduction) in collagen type I expression compared to the average gene expression in placebo and HA groups (average gene expression 38.9 and 31.32 respectively; Fig. $3 \mathrm{~L}$ ). Regarding the control cartilage samples, there was no relative changes in gene expression compared to sham, with the exception of an increase in collagen type II expression in $30 \mathrm{~g}$ and $100 \mathrm{~g}$ sprifermin $(p=0.001$ and $p=0.055$ respectively; data not shown).

\section{Results: Secondary Endpoints}

In order to assess relevant clinical measurements and more functional outcomes, we performed as secondary endpoint measurements the following: clinical assessments including lameness and flexion tests, gross joint evaluations, synovial fluid analysis, arthroscopic and radiographic imaging. 


\section{Clinical Assessments}

All animals recovered from general anesthesia without incident. One animal (Horse \# 4) had an episode of abdominal discomfort after second look arthroscopy that required surgical management. She subsequently developed grade 2 gastric ulcers and required omeprazole therapy thereafter for one month. Horses \#8 and \#17 were lame enough post-operatively that a longer period stall rest and phenylbutazone were given.

For lameness and flexion assessment, looking at week by week results and combining scores from all sprifermin treated animals, scores peaked in the early postoperative phase and again after the second look arthroscopy at week twelve. This secondary peak was more prominent in placebo treated group (Fig. S2A and B). In addition, comparing sprifermin to HA treatment, scores from sprifermin treated joints were closer to sham values (Fig. S2A and B). We observed a gradual improvement in lameness and flexion test scores and at time points after week 14 , improvement in scores reached statistical significance $(p<0.05$; Fig. S2C and D). When we analyzed the lameness sum scores for the whole duration of this study, it was seen that no single dose of sprifermin was superior to placebo treatment (Fig. 4A), but the combined results of three doses of sprifermin showed a significant improvement in lameness scores $(2.5 \pm 3.777$ and $6.30 \pm 5.51, p=0.046$; Fig. 4B). In the matter of flexion test, we observed a near to significant improvement when we combined results from various sprifermin dose treatment groups (sprifermin 3.389 \pm 6.48 , placebo $7.667 \pm 6.851, p=0.053$; Fig. 4D).

\section{Synovial Fluid Analysis}

Synovial fluid was obtained from all horses at all time points without complication. There was no effect of treatment with sprifermin, HA or placebo on total synovial fluid white cell counts or total protein (Fig. S2E and F).

\section{Second Look Arthroscopy and Gross Evaluation}

Twelve weeks post-surgery, arthroscopic evaluation demonstrated a significant improvement in cartilage defect healing in joints treated with sprifermin compared to joints treated with $\mathrm{HA}$ or placebo (sprifermin $10.19 \pm 2.71$ versus placebo $9.16 \pm 2.93, p=0.02$, Fig. $5 B$ ). There was no significant difference between groups when looking at individual doses of sprifermin or between $\mathrm{HA}$ and placebo treated groups (Fig. 5A). Evaluation of sub-scores showed significantly superior scores in surface texture $(p=0.05)$ of sprifermin treated joints (Fig. S3F). In addition, treatment with $10 \mathrm{~g}, 30 \mathrm{~g}$ or $100 \mathrm{~g}$ sprifermin demonstrated a higher benefit when compared to HA treatment $(37.6 \%, 24.7 \%$ and $34.1 \%$ respectively versus $17.6 \%)$.

Post-mortem evaluation revealed that treatment with no single dose of sprifermin resulted in a significant improvement in overall gross morphology (Fig. $5 \mathrm{C}$ ). When we combined results from various sprifermin treatment groups, a near to significant improvement was detected $(22.42 \pm 3.40$ versus $20.03 \pm 3.09, p=0.060$; Fig. 5D). In analysis of multiple sub-scores, we detected a significant $(p=0.039)$ and a near to significant $(p=$ 0.058 ) improvement in cartilage surface and defect fill respectively (Fig. $S 4 \mathrm{I}$ and $\mathrm{K}$ ).

We detected no significant difference between HA and placebo treated limbs. The percentage benefit evaluation showed a larger and inversely dose related benefit in sprifermin treatment compared to HA (ranged from $37.4 \%$ 


\section{Radiographic and Computed Tomography Scores}

Radiographs and CT examination showed decreased osteoarthritic changes in sprifermin treated joints compared to placebo and HA (Fig. 6).

At week 12 , radiography scores were significantly improved in high dose $(100 \mathrm{~g})$ sprifermin $(p<0.05)$ or when we combined the scores from three sprifermin dose-treatment groups $(\mathrm{p}<0.01$; Fig. $6 \mathrm{~B}$ and $\mathrm{C})$. The same significant improvement in radiographic scores of the $100 \mathrm{~g}$ group was detected at week 24 post-surgery $(p=0.016$; Fig. $6 \mathrm{D}$ ) and combined sprifermin scores were also significantly improved ( $8.31 \pm 0.941$ versus $17.39 \pm 0.883, p=$ 0.011; Fig. 6E). Peri-articular soft tissue swelling and soft tissue mineralization were the main radiographic features improved as a result of sprifermin treatment ( $p=0.024$ and 0.064 respectively (Fig. S5E and F). There was an increased benefit in low dose (10 g) and high dose (100 g) sprifermin compared to HA (48.6\% and 70\% respectively versus $25.7 \%$ ).

At week 24, CT scores of combined sprifermin showed significantly less osteoarthritic change compared to placebo $(20.39 \pm 2.38$ versus $18.53 \pm 2.09, p=0.043$; Fig. $6 \mathrm{H})$. Evaluation of sub-scores showed significantly reduced osteophyte development in sprifermin treated limbs at 24 weeks $(p<0.01)$ compared to HA and placebo treated joints (Fig. S 6G). Additionally, treatment with any of three doses of sprifermin had a larger benefit when compared to HA (ranged from $26.3 \%$ to $36.4 \%$ versus $3.3 \%$ ).

\section{Discussion}

Development of DMOADs continues to be a challenge in orthopedics; many agents have failed in clinical trials, and osteoarthritis remains a profound clinical problem with few positive interventions outside of pain management and eventual joint replacement. In human clinical studies, sprifermin has been shown to be effective and safe [32,33]. Further investigation in a large animal model is a critical step in evaluating sprifermin as a potential DMOAD since more controlled and early disease can be evaluated. In the present study, using an equine animal model we demonstrated that applying sprifermin in conjunction of microfracture treatment can improve the outcome of the surgery. This result is consistent with in vivo findings in other animal models and in vitro and ex vivo studies that suggest an anabolic role for sprifermin in cartilage healing and hemostasis [21, 29, 30, 34, 43].

Comparing various animal models, horses are considered an excellent model for articular cartilage studies [35, 36]. Poor intrinsic regenerative capacity, large easily sampled joints, and thick articular cartilage in horses make their articular cartilage defects comparable to the same lesions in humans and allow comprehensive cartilage studies to test efficacy and safety of new cartilage treatments prior to human clinical trials. In addition, horses like humans suffer from sport injuries and as a result articular cartilage injury treatments and surgeries are better understood in horses than other animal models [44].

In this study, we examined the impact of sprifermin in both functional and structural components of cartilage healing. In sprifermin treated joints, we detected a significant improvement in lameness; arthroscopic evaluation showed superior quality and quantity of the repair tissue; and both radiographic and CT scan imaging showed 
mitigated pathology. In post-mortem evaluation of the repair tissue, we detected an improvement in the gross morphology of the defect site, the overall histological assessment of the kissing region, and the expression of the constitutive genes. For the most part, no single dose of sprifermin showed a statistically significant advantage over placebo or HA. However, when we combined scores from three dose-treatment groups, changes were significant. In addition, looking closely at our non-significant changes in sprifermin scores, we observed that in most cases at least $50-60 \%$ of subjects showed an improvement compared to the average placebo scores. This lack of significance can be due to the small per group sample size $(n=6)$ that is a known limitation when using an equine model [36]. We compared the outcome of the sprifermin treatment to HA treatment, as a common osteoarthritis intraarticular therapeutics, and the majority of tests detected higher benefit associated with sprifermin.

Through our ante-mortem and post-mortem tests, we observed a concentration dependent benefit in sprifermin treatment with highest benefit in $100 \mathrm{~g}$ injections with the exception of our lameness and flexion test scores. Increased lameness in high dose sprifermin group may be a consequence of an acute and transient inflammatory reaction (joint stiffness and arthralgia) that was previously observed in human clinical trials with administration of high dose sprifermin (100 or $300 \mathrm{~g}$ ) [31, 33]. Another limitation was observed in our early clinical assessment. For purpose of this study, horses were not randomized based on surgical status. As a result our placebo cohort had a slight higher lameness (not statistically significant) immediately post-surgery that was revealed to us at the end of the study after unblinding. Lameness and flexion test scores were not adjusted to the baseline. At later time points, the slight initial dissimilarity expanded and we detected a significant improvement in sprifermin treated joints.

In addition to efficiency of sprifermin on improvement of cartilage healing, this study, notably, detected no adverse effects following sprifermin treatment. This finding is also consistent with approved safety of sprifermin during human clinical trials [31, 33]. We also detected that the unique three intermittent intraarticular sprifermin injections alter gene expression in chondrocytes (e.g. increase in type II collagen and aggrecan expression and decrease in collagen type I expression) at the repair site and areas in direct contact with it. We assume that such a response is mediated through sprifermin binding to its functional receptor, FGFR3 and activation of ERK1/2 signaling pathways. It has been shown that blockade of this pathway can result in inhibition of the sprifermin impact $[22,29]$. Previous studies have suggested the potential benefit of FGF18FGFR3 binding and an increase in FGFR3 expression on improvement of articular cartilage integrity $[45,46]$. Notably, we detected an increase in average FGFR3 gene expression in all three regions of interest following 100 $\mathrm{g}$ sprifermin treatment. Although increase in gene expression was not statistically significant, 5 animals out of 6 showed improvement in FGFR3 expression in peri-lesion and kissing region when compared to the average gene expression in placebo group (Fig S6, I and J).

Although for an equine study this study was conducted with large number of subjects but it still was likely underpowered. While this was a considerably protracted evaluation timeframe, larger studies with higher number of subjects and even longer durations, will be critical to investigate sprifermin's long term effect on cartilage healing and functional restoration. Wilke et al. recommend at least 8 months to assess the outcome of a treatment in an equine cartilage defect model [47].

In spite of such limitations, this study clearly shows how human clinical trials can be enhanced by large animal data where we have better control over injury and early intervention and we are able to conduct post-mortem

Page $11 / 24$ 
evaluations. Future in vivo studies will be needed to understand the biomechanical benefits of sprifermin treatment and investigate the underlying mechanism of sprifermin effect on cartilage healing and clinically relevant function.

\section{Conclusion}

This study shows for the first time that intra-articular sprifermin enhances the effects of micro-fracture surgery in an equine model and identifies potential benefit from sprifermin treatment in early cartilage injuries in both veterinary and clinical medicine. Showing both functional and structural effect of sprifermin treatment, this study represents an advancement in cartilage injury and osteoarthritis therapeutic development.

\section{Supplementary File Legends}

Additional File 1 (Table S1) Second look arthroscopy scoring. Arthroscopic images were scored for three different metrics on a scale of 1 to 5. Scoring system was adopted from Van den Borne et al., 2007 and Goebel et al., 2012 [38,39].

Additional File 2 (Table S2) Gross morphology scoring. Gross morphology images were scored for six metrics on a scale of 1 to 5 based on OARSI scoring system $[38,40]$.

Additional File 3 (Table S3) Radiography scoring. Radiographic images were scored for four metrics on a scale of 1 to 5 . Scoring system was adapted from Boyce et al, 2013 [41].

Additional File 4 (Table S4) CT Scan scoring. CT Scan images were scored for six metrics on a scale of 1 to 5. Scoring system was adapted from Cohen et al., 2015 [42].

Additional file 5 (Fig. S1) Histologic evaluation of kissing region and chondrocyte proliferation assessment. Safranin O/ fast Green stained slides of kissing region were scored for ten metrics: (A) matrix staining, (B) cellularity, (C) superficial zone clustering, (D) middle-deep zone clustering, $(E)$ surface architecture, $(F)$ subchondral bone involvement, (G) lesion intensity, (H) superficial zone assessment, (I) middle-deep zone assessment, and $(\mathrm{J})$ overall assessment. $(K)$ chondrocytes numbers were counted in control region, $(\mathrm{L})$ lesion, and $(\mathrm{M})$ kissing region of fetlocks. $\mathrm{n}=6$ or $18,{ }^{\star} p<0.05,{ }^{\star \star} p<0.01$ by un-paired Mann-Whitney test.

Additional File 6 (Fig. S2) Clinical assessment and synovial fluid analysis. (A) Scores of ten lameness assessments from different treatment groups showed an improvement in combined sprifermin group at late time points. (B) Scores from ten flexion tests from different treatment groups showed an improvement in sprifermin group at late time points. (C) Comparing combined lameness scores from three sprifermin groups with placebo group and running multiple non-parametric t-tests showed a significant improvement in lameness scores at week $14(p=0.019), 18(0.004), 22(p=0.005)$ and $24(p=0.028)$. (D) Flexion test scores showed similar improvement at week $18(p=0.009), 22(p=0.004)$, and 24( $p=0.046)$. (E) White cell counts after start of the intraarticular injections (week 4 to 24 ) did not detect any significant difference between groups. (F) Total protein amount in synovial fluid at multiple time points did not show any significant difference between groups. Dotted line indicates median of the placebo group. $\mathrm{n}=6$ or $18,{ }^{*} p<0.05$ by Mann-Whitney test ( $\mathrm{C}$ and $\mathrm{D}$ ) and KruskalWallis and Dunn's multiple comparison test ( $\mathrm{E}$ and $\mathrm{F})$. 
Additional File 7 (Fig. S3) Arthroscopic images of the defect site at 12 weeks post-surgery were evaluated and scored for three sub-scores. (A) percentage of the defect fill, (B) color of the repair tissue, (C) surface of the repair tissue. (D to F) Scores from three doses of sprifermin combined to improve the statistical power. Dotted line indicates median of the placebo group. For $A$ to $C, n=6$ or 18 , and for $D$ to $F n=18, * p<0.05$ by KruskalWallis and Dunn's multiple comparison test.

Additional File 8 (Fig. S4) Gross morphology of the defect site was evaluated and scored for 6 sub-scores. (A) synovial membrane appearance, (B) Palmar/ Plantar condyle appearance, (C) percentage of the defect fill, (D) color of repair tissue, $(E)$ surface of the repair tissue, and $(F)$ osteophyte formation. ( $G$ to $L$ ) scores from three doses of sprifermin combined to improve the statistical power. Dotted line indicates median of the placebo group. For $\mathrm{A}$ to $\mathrm{F}, \mathrm{n}=6$ or 18 , for $\mathrm{G}$ to $\mathrm{L}, \mathrm{n}=18$, ${ }^{\star} p<0.05$ by Kruskal-Wallis and Dunn's multiple comparison test.

Additional File 9 (Fig. S5) Radiographic images of fetlocks at 24 weeks post-surgery were scored for four subscores. (A) peri-articular soft tissue swelling, (B) soft tissue mineralization, (C) formation of osteophytes, and (D) formation of enthesophytes. $(\mathrm{E}$ to $\mathrm{H})$ Scores from three doses of sprifermin combined to improve the statistical power. Dotted line indicates median of the placebo group. For $A$ to $D, n=6$ or 18 , for $E$ to $H, n=18$. ${ }^{*} p<0.05$ by Kruskal-Wallis and Dunn's multiple comparison test.

Additional File 10 (Fig. S6) Post-mortem CT-Scan images were evaluated and scored for four sub-scores; and FGFR3 expression was measured in three regions. (A) sclerosis of the lateral condyle, (B) Sclerosis of lateral proximal sesamoid bone, $(C)$ osteophyte, and $(D)$ enthesophyte formation. $(E$ to $H)$ Scores from three doses of sprifermin combined to improve the statistical power. (I) FGFR3 gene expression was measured in peri-lesion (I) and kissing region $(\mathrm{J})$ of fetlocks treated with $100 \mathrm{mg}$ sprifermin, HA, or placebo. Dotted line indicates median of the placebo group. For $A$ to $D, n=6$ or 18 and for $E$ to $H, n=18$, ${ }^{*} p<0.05$ by Kruskal-Wallis and Dunn's multiple comparison test.

\section{Abbreviations}

ACTB: actin beta; B2M: beta-2 microglobulin; BMP: Bone morphogenic protein; cDNA: complementary deoxyribonucleic acid; COMP: cartilage oligomeric matrix protein; CT scan: Computed Tomography scan ; CT: cycle threshold; DLPMO: dorsolateral/palmaromedial oblique; DMOADs: Disease Modifying Osteoarthritis Drugs; DMPLO: dorsomedial/palmarolateral oblique; ERK: extracellular receptor kinase; FGF18: fibroblast growth factor-18; FGFR3: fibroblast growth factor receptor 3; GAPDH: glyceraldehyde 3-phosphate dehydrogenase; GUSB: glucuronidase beta; HA: Hyaluronan; LFTC: lateral femorotibial compartment; MFTC: medial femorotibial compartments; MFX: microfracture; MRI: magnetic resonance imaging; PCR: polymerase chain reaction; PRP: Platelet-rich plasma; PTOA: post-traumatic osteoarthritis ; RNA: ribonucleic acid; SDHA: succinate dehydrogenase complex flavoprotein subunit A; TFTJ: total femorotibial joint ; TGFb: transforming growth factor beta; TP: total protein; WBC: white blood cell

\section{Declarations}

\section{Acknowledgments}


The authors gratefully acknowledged the advice and technical assistance of Mr. Ryan Smalley, Mr. Patrick Diviney, Dr. Bhavana Monhanraj.

\section{Authors' Contributions}

All authors contributed to the design (except M.G. and H.G.) and execution (except H.G.) and data interpretation of this study. All authors contributed to the writing and editing of this manuscript (except M.G.).

\section{Funding}

This work was supported by a grant from Merck KGaA. Additional support was provided by the Department of Veteran's Affairs (I01 RX001213 and VA RR\&D I01 RX001321) and the NIH-NIAMS via R01 AR071340 (GRD), and the Penn Center for Musculoskeletal Diseases Biomechanics and Histology Core (NIH NIAMS P30-AR069619).

\section{Availability of data and materials}

The datasets used and/or analyzed during the current study are available from the corresponding author on reasonable request.

\section{Ethics approval and consent to participate}

The study was conducted with all necessary compliance requirements and was approved by the University of Pennsylvania Animal Care and Use Committee.

\section{Consent for publication}

not applicable

\section{Competing interests}

Hans Guehring, one of the authors, is a paid employee of Merck KGaA.

\section{References}

1. Brown TD, Johnston RC, Saltzman CL, Marsh JL, Buckwalter JA. Posttraumatic osteoarthritis: a first estimate of incidence, prevalence, and burden of disease. J Orthop Trauma. 2006;20(10):739-44.

2. Showery JE, Kusnezov NA, Dunn JC, Bader JO, Belmont PJ, Jr., Waterman BR. The Rising Incidence of Degenerative and Posttraumatic Osteoarthritis of the Knee in the United States Military. J Arthroplasty. 2016;31(10):2108-14.

3. U.S. Centers for Disease Control and Prevention. Arthritis in America: Time to Take Action! 2017 [Available from: https://www.cdc.gov/vitalsigns/pdf/2017-03-vitalsigns.pdf.

4. Osteoarthritis Research Society International (OARSI). OA as a Serious Disease 2016 [Available from: https://www.oarsi.org/sites/default/files/docs/2016/oarsi_white_paper_oa_serious_disease_121416_1.pdf.

5. Steadman JR, Briggs KK, Rodrigo JJ, Kocher MS, Gill TJ, Rodkey WG. Outcomes of microfracture for traumatic chondral defects of the knee: average 11-year follow-up. Arthroscopy. 2003;19(5):477-84. 
6. Steadman JR, Rodkey WG, Briggs KK. Microfracture: Its History and Experience of the Developing Surgeon. Cartilage. 2010;1(2):78-86.

7. Breinan HA, Martin SD, Hsu HP, Spector M. Healing of canine articular cartilage defects treated with microfracture, a type-Il collagen matrix, or cultured autologous chondrocytes. J Orthop Res. 2000;18(5):7819.

8. Mithoefer K, McAdams T, Williams RJ, Kreuz PC, Mandelbaum BR. Clinical efficacy of the microfracture technique for articular cartilage repair in the knee: an evidence-based systematic analysis. Am J Sports Med. 2009;37(10):2053-63.

9. Oussedik S, Tsitskaris K, Parker D. Treatment of articular cartilage lesions of the knee by microfracture or autologous chondrocyte implantation: a systematic review. Arthroscopy. 2015;31(4):732-44.

10. Furukawa T, Eyre DR, Koide S, Glimcher MJ. Biochemical studies on repair cartilage resurfacing experimental defects in the rabbit knee. J Bone Joint Surg Am. 1980;62(1):79-89.

11. Knutsen G, Engebretsen L, Ludvigsen TC, Drogset JO, Grontvedt T, Solheim E, et al. Autologous chondrocyte implantation compared with microfracture in the knee. A randomized trial. J Bone Joint Surg Am. 2004;86(3):455-64.

12. Camarero-Espinosa $S$, Rothen-Rutishauser B, Foster EJ, Weder C. Articular cartilage: from formation to tissue engineering. Biomater Sci. 2016;4(5):734-67.

13. Gaugler M, Wirz D, Ronken S, Hafner M, Gopfert B, Friederich NF, et al. Fibrous cartilage of human menisci is less shock-absorbing and energy-dissipating than hyaline cartilage. Knee Surg Sports Traumatol Arthrosc. 2015;23(4):1141-6.

14. Kang SW, Bada LP, Kang CS, Lee JS, Kim CH, Park JH, et al. Articular cartilage regeneration with microfracture and hyaluronic acid. Biotechnol Lett. 2008;30(3):435-9.

15. Kuo AC, Rodrigo JJ, Reddi AH, Curtiss S, Grotkopp E, Chiu M. Microfracture and bone morphogenetic protein 7 (BMP-7) synergistically stimulate articular cartilage repair. Osteoarthritis Cartilage. 2006;14(11):1126-35.

16. Guney A, Akar M, Karaman I, Oner M, Guney B. Clinical outcomes of platelet rich plasma (PRP) as an adjunct to microfracture surgery in osteochondral lesions of the talus. Knee Surg Sports Traumatol Arthrosc. 2015;23(8):2384-9.

17. Yang HS, La WG, Bhang SH, Kim HJ, Im GI, Lee H, et al. Hyaline cartilage regeneration by combined therapy of microfracture and long-term bone morphogenetic protein-2 delivery. Tissue Eng Part A. 2011;17(1314):1809-18.

18. Solheim E, Hegna J, Inderhaug E, Oyen J, Harlem T, Strand T. Results at 10-14 years after microfracture treatment of articular cartilage defects in the knee. Knee Surg Sports Traumatol Arthrosc. 2016;24(5):158793.

19. Ohbayashi N, Shibayama M, Kurotaki Y, Imanishi M, Fujimori T, Itoh N, et al. FGF18 is required for normal cell proliferation and differentiation during osteogenesis and chondrogenesis. Genes Dev. 2002;16(7):870-9.

20. Liu Z, Lavine KJ, Hung IH, Ornitz DM. FGF18 is required for early chondrocyte proliferation, hypertrophy and vascular invasion of the growth plate. Dev Biol. 2007;302(1):80-91.

21. Ellsworth JL, Berry J, Bukowski T, Claus J, Feldhaus A, Holderman S, et al. Fibroblast growth factor-18 is a trophic factor for mature chondrocytes and their progenitors. Osteoarthritis Cartilage. 2002;10(4):308-20. 
22. Davidson D, Blanc A, Filion D, Wang H, Plut P, Pfeffer G, et al. Fibroblast growth factor (FGF) 18 signals through FGF receptor 3 to promote chondrogenesis. J Biol Chem. 2005;280(21):20509-15.

23. Correa D, Somoza RA, Lin P, Greenberg S, Rom E, Duesler L, et al. Sequential exposure to fibroblast growth factors (FGF) 2, 9 and 18 enhances hMSC chondrogenic differentiation. Osteoarthritis Cartilage. 2015;23(3):443-53.

24. Naski MC, Colvin JS, Coffin JD, Ornitz DM. Repression of hedgehog signaling and BMP4 expression in growth plate cartilage by fibroblast growth factor receptor 3. Development. 1998;125(24):4977-88.

25. Krejci P, Krakow D, Mekikian PB, Wilcox WR. Fibroblast growth factors 1, 2, 17, and 19 are the predominant FGF ligands expressed in human fetal growth plate cartilage. Pediatr Res. 2007;61(3):267-72.

26. Ellman MB, Yan D, Ahmadinia K, Chen D, An HS, Im HJ. Fibroblast growth factor control of cartilage homeostasis. J Cell Biochem. 2013;114(4):735-42.

27. Barr L, Getgood A, Guehring H, Rushton N, Henson FM. The effect of recombinant human fibroblast growth factor-18 on articular cartilage following single impact load. J Orthop Res. 2014;32(7):923-7.

28. Moore EE, Bendele AM, Thompson DL, Littau A, Waggie KS, Reardon B, et al. Fibroblast growth factor-18 stimulates chondrogenesis and cartilage repair in a rat model of injury-induced osteoarthritis. Osteoarthritis Cartilage. 2005;13(7):623-31.

29. Gigout A, Guehring H, Froemel D, Meurer A, Ladel C, Reker D, et al. Sprifermin (rhFGF18) enables proliferation of chondrocytes producing a hyaline cartilage matrix. Osteoarthritis Cartilage. 2017;25(11):1858-67.

30. Sennett ML, Meloni GR, Farran AJE, Guehring H, Mauck RL, Dodge GR. Sprifermin treatment enhances cartilage integration in an in vitro repair model. J Orthop Res. 2018;36(10):2648-56.

31. Lohmander LS, Hellot S, Dreher D, Krantz EF, Kruger DS, Guermazi A, et al. Intraarticular sprifermin (recombinant human fibroblast growth factor 18) in knee osteoarthritis: a randomized, double-blind, placebo-controlled trial. Arthritis Rheumatol. 2014;66(7):1820-31.

32. Hochberg MC, Guermazi A, Guehring H, Aydemir A, Wax S, Fleuranceau-Morel P, et al. Effect of Intra-Articular Sprifermin vs Placebo on Femorotibial Joint Cartilage Thickness in Patients With Osteoarthritis: The FORWARD Randomized Clinical Trial. JAMA. 2019;322(14):1360-70.

33. Dahlberg LE, Aydemir A, Muurahainen N, Guhring H, Fredberg Edebo H, Krarup-Jensen N, et al. A first-inhuman, double-blind, randomised, placebo-controlled, dose ascending study of intra-articular rhFGF18 (sprifermin) in patients with advanced knee osteoarthritis. Clin Exp Rheumatol. 2016;34(3):445-50.

34. Power J, Hernandez P, Guehring H, Getgood A, Henson F. Intra-articular injection of rhFGF-18 improves the healing in microfracture treated chondral defects in an ovine model. J Orthop Res. 2014;32(5):669-76.

35. Mcllwraith CW, Fortier LA, Frisbie DD, Nixon AJ. Equine Models of Articular Cartilage Repair. Cartilage. 2011;2(4):317-26.

36. Chu CR, Szczodry M, Bruno S. Animal models for cartilage regeneration and repair. Tissue Eng Part B Rev. 2010;16(1):105-15.

37. American Association of Equine Practitioners (AAEP). Lameness Exams: Evaluating the Lame Horse [Available from: https://aaep.org/horsehealth/lameness-exams-evaluating-lame-horse.

38. van den Borne MP, Raijmakers NJ, Vanlauwe J, Victor J, de Jong SN, Bellemans J, et al. International Cartilage Repair Society (ICRS) and Oswestry macroscopic cartilage evaluation scores validated for use in

Page $16 / 24$ 
Autologous Chondrocyte Implantation (ACI) and microfracture. Osteoarthritis Cartilage. 2007;15(12):1397402.

39. Goebel L, Orth P, Muller A, Zurakowski D, Bucker A, Cucchiarini M, et al. Experimental scoring systems for macroscopic articular cartilage repair correlate with the MOCART score assessed by a high-field MRI at 9.4 T--comparative evaluation of five macroscopic scoring systems in a large animal cartilage defect model. Osteoarthritis Cartilage. 2012;20(9):1046-55.

40. Little CB, Smith MM, Cake MA, Read RA, Murphy MJ, Barry FP. The OARSI histopathology initiative recommendations for histological assessments of osteoarthritis in sheep and goats. Osteoarthritis Cartilage. 2010;18 Suppl 3:S80-92.

41. Boyce MK, Trumble TN, Carlson CS, Groschen DM, Merritt KA, Brown MP. Non-terminal animal model of post-traumatic osteoarthritis induced by acute joint injury. Osteoarthritis Cartilage. 2013;21(5):746-55.

42. Cohen MM, Vela ND, Levine JE, Barnoy EA. Validating a new computed tomography atlas for grading ankle osteoarthritis. J Foot Ankle Surg. 2015;54(2):207-13.

43. Howard D, Wardale J, Guehring H, Henson F. Delivering rhFGF-18 via a bilayer collagen membrane to enhance microfracture treatment of chondral defects in a large animal model. J Orthop Res. 2015;33(8):1120-7.

44. Frisbie DD, Cross MW, Mcllwraith CW. A comparative study of articular cartilage thickness in the stifle of animal species used in human pre-clinical studies compared to articular cartilage thickness in the human knee. Vet Comp Orthop Traumatol. 2006;19(3):142-6.

45. Valverde-Franco G, Binette JS, Li W, Wang H, Chai S, Laflamme F, et al. Defects in articular cartilage metabolism and early arthritis in fibroblast growth factor receptor 3 deficient mice. Hum Mol Genet. 2006;15(11):1783-92.

46. Tang J, Su N, Zhou S, Xie Y, Huang J, Wen X, et al. Fibroblast Growth Factor Receptor 3 Inhibits Osteoarthritis Progression in the Knee Joints of Adult Mice. Arthritis Rheumatol. 2016;68(10):2432-43.

47. Wilke MM, Nydam DV, Nixon AJ. Enhanced early chondrogenesis in articular defects following arthroscopic mesenchymal stem cell implantation in an equine model. J Orthop Res. 2007;25(7):913-25.

\section{Figures}


A

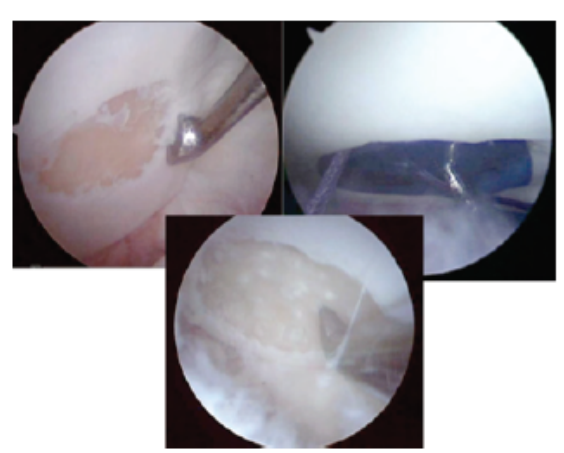

B

Four Fetlocks:

- Sham

- MFX + Saline Injection (Placebo)

- MFX + Hyaluronan (HA) Injection

- MFX + One of three doses of sprifermin $(10,30$, or $100 \mu \mathrm{l})$

C

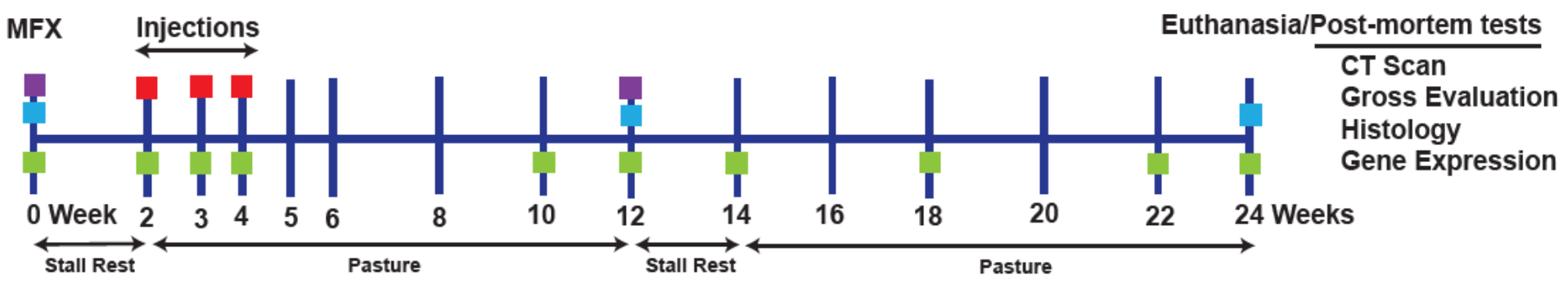

Injections (Sprifermin, HA, or Saline)

Arthroscopy

Radiography

Lameness and Flexion Test

Figure 1

Surgical technique, study design, and study time line (A) A $15 \mathrm{~mm}$ circumferential full thickness articular cartilage defect was created on the central dorsal aspect of the lateral proximal sesamoid bone (left). Radiographic film was used as a template to ensure the defects were of uniform size (right) and an awl was used to micropick 25-30 locations within the defect (bottom). (B) Four fetlocks of each animal were randomly assigned to one of the treatment groups. (C) Schematic overview of the study time line. MFX= microfracture treatment, $\mathrm{HA}=$ hyaluronan. 
A

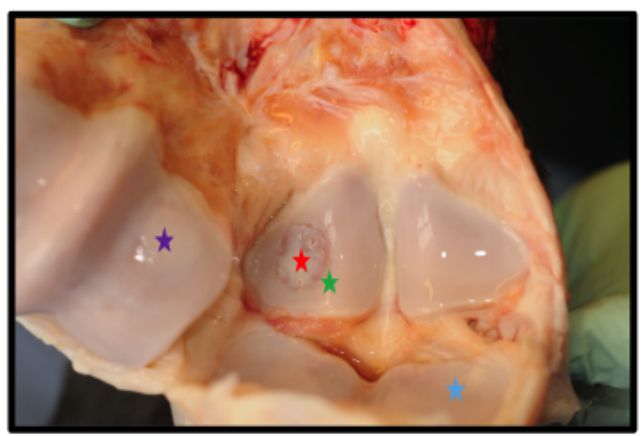

Lesion $\$$ Peri-lesion $\$$ Kissing Region $\$$ Control

c

Control

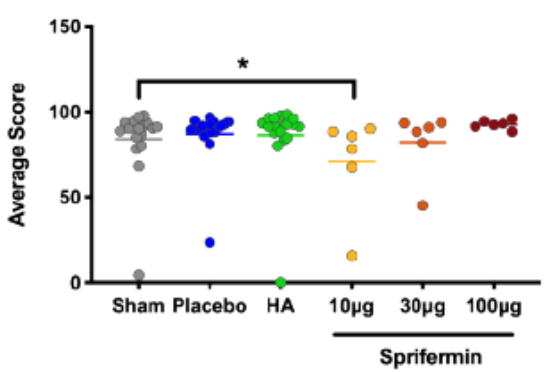

B

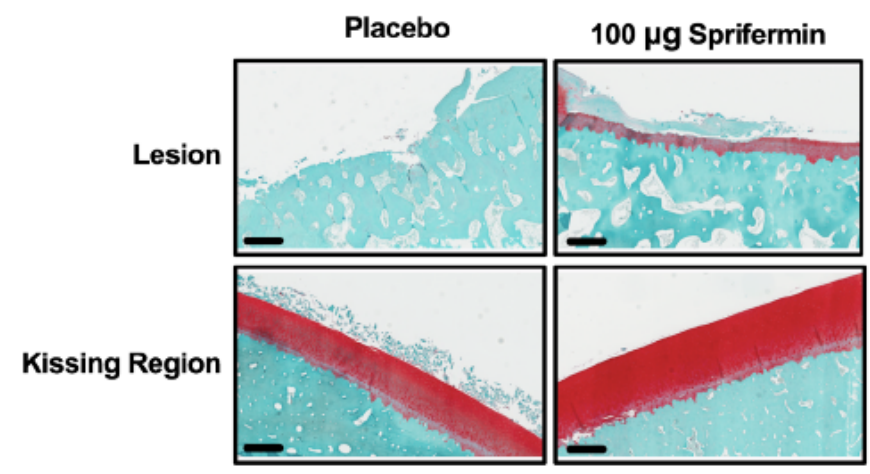

E

Kissing Region

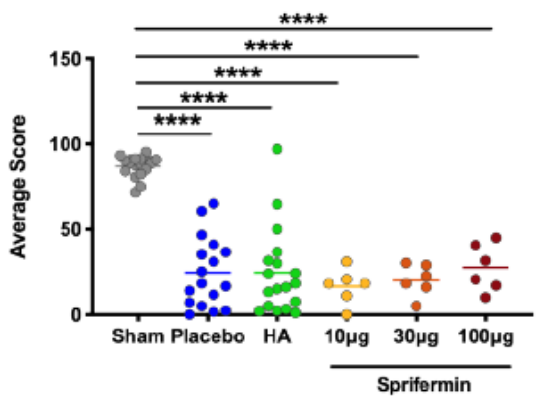

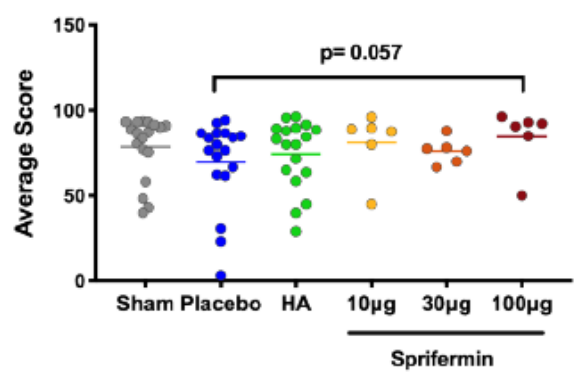

Figure 2

Histological Evaluation of fetlocks (A) Cartilage samples were harvested from four regions of interest inside fetlocks. (B) An example of the Safranin O/Fast Green staining in lesion and kissing region of placebo and 100 $\mu \mathrm{g}$ sprifermin treated fetlocks of an individual horse. 20x magnification images, scale bar $=500 \mu \mathrm{m}$. (C) Overall assessment of control, (D) Lesion, and (E) Kissing region compares scores in joints received HA or sprifermin treatment with sham and placebo treated joints. $n=6$ or $18,{ }^{*} p<0.05$ by un-paired Mann-Whitney test. 
A

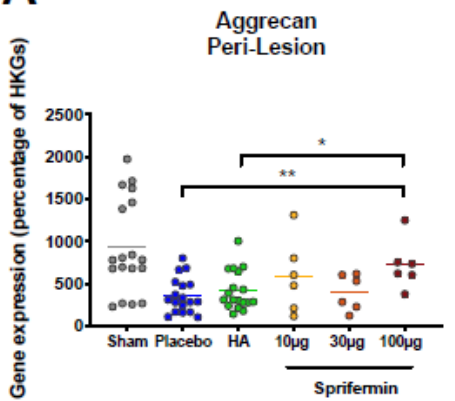

E
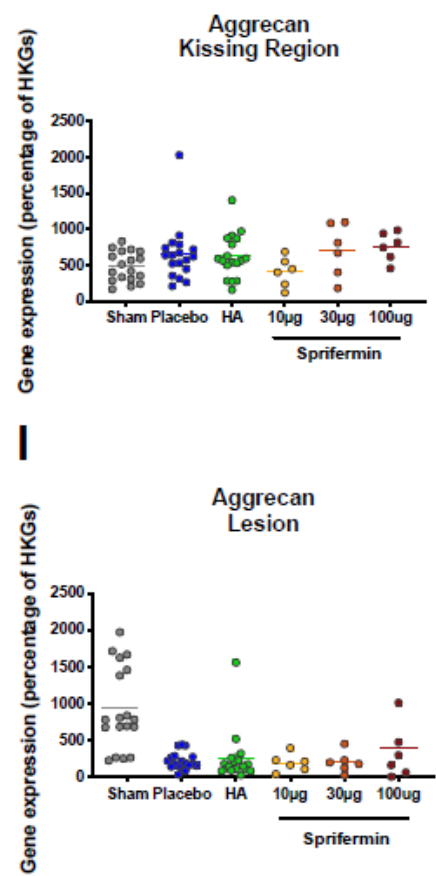

B

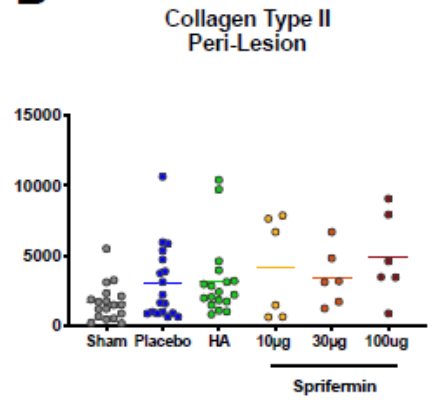

F

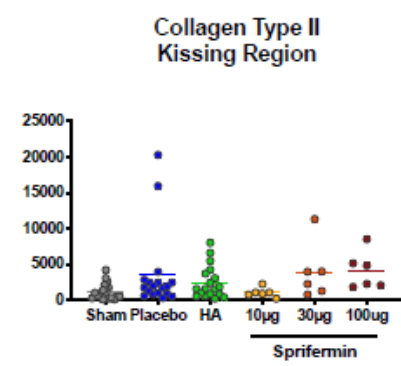

J

Collagen Type II

Lesion

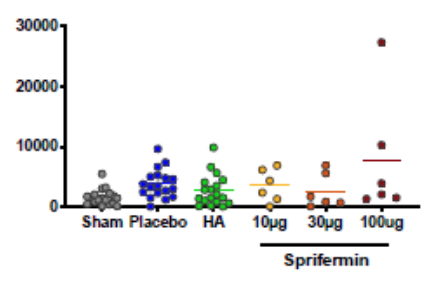

C

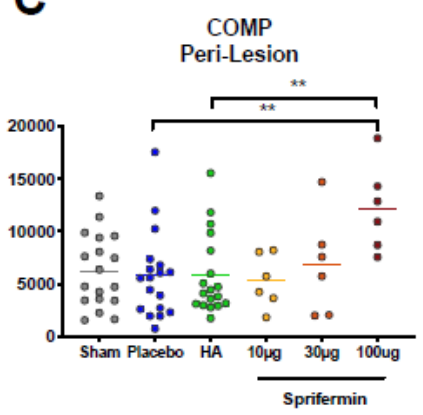

G

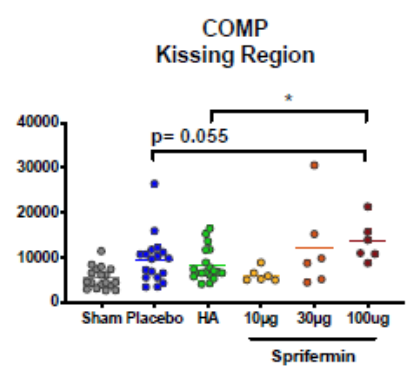

K

COMP
Lesion

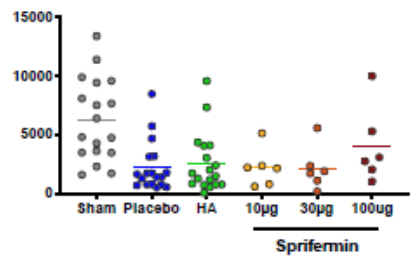

D

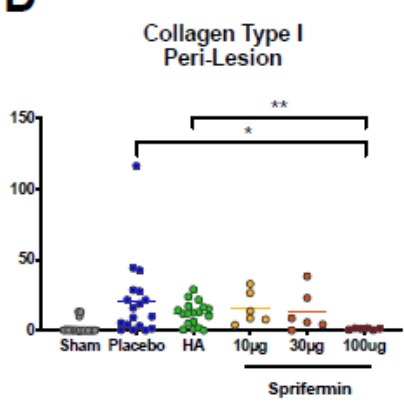

H

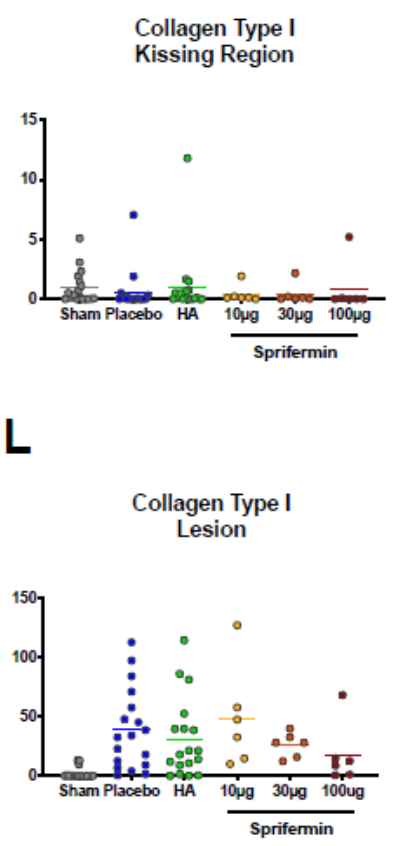

Figure 3

Evaluation of gene expression in three regions of interest based on post-mortem Real-Time PCR results (A to D) Aggrecan, Collagen type II, COMP, and Collagen type I expression in peri-lesion showed dose response changes in gene expression following sprifermin treatment. $(\mathrm{E}$ to $\mathrm{H}$ ) Changes in gene expression in kissing region showed a significant and near to significant improvement in COMP expression. (I to L) Changes in gene expression in lesion showed a non-significant dose response to sprifermin treatment. Gene expression in sprifermin treated joints were compared to placebo or HA treatment groups using un-paired Mann-Whitney test. $n=6$ or 18 , ${ }^{*} \mathrm{p}<0.05,{ }^{* *} \mathrm{p}<0.01$ 
A

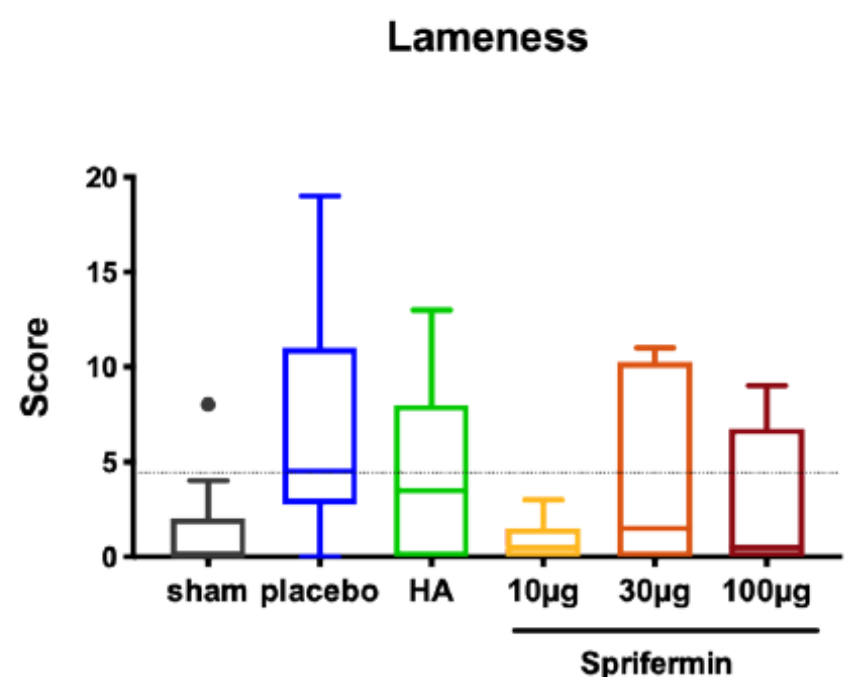

C

Flexion Test

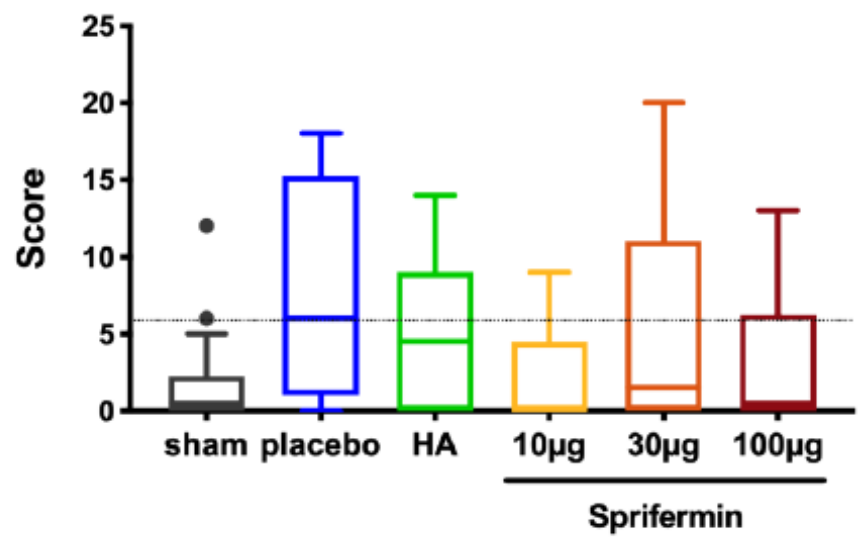

B
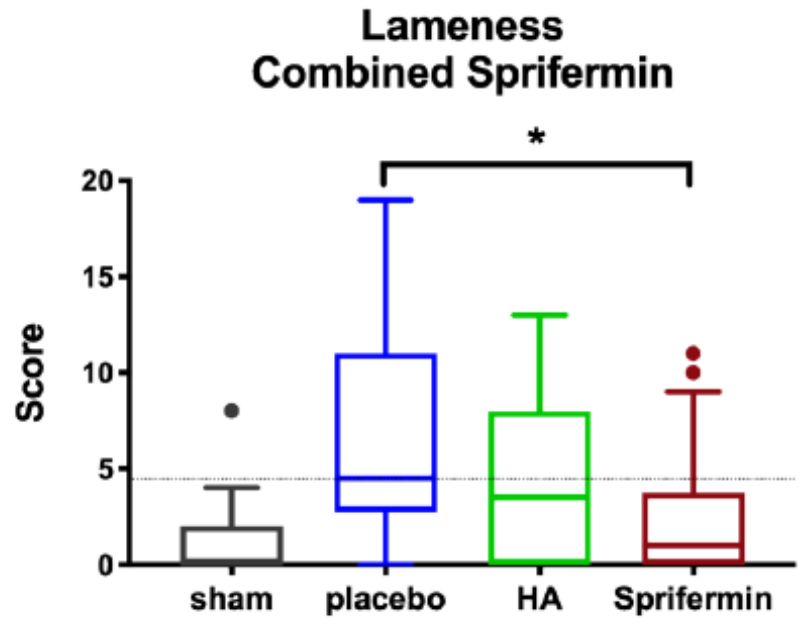

D

Flexion Test

Combined Sprifermin

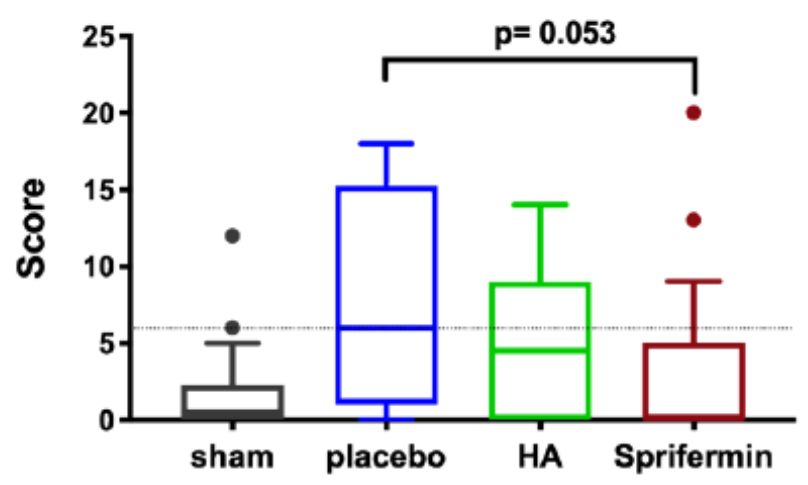

Figure 4

Clinical assessment via lameness and flexion tests. (A) An average of ten lameness scores for limbs treated with various doses of sprifermin, HA, or placebo. (B) Combined scores from three sprifermin dose-treatment groups showed a significant reduction in lameness when compared to the placebo. (C) An average of ten flexion test scores for limbs received various intraarticular treatments. (D) Combined scores from three dose-treatment groups showed near to significant reduction compared to the placebo. Dotted line indicates median of the placebo group. $\mathrm{n}=6$ or $18,{ }^{*} \mathrm{p}<0.05$ by Kruskal-Wallis and Dunn's multiple comparison test. 
A

Arthroscopy

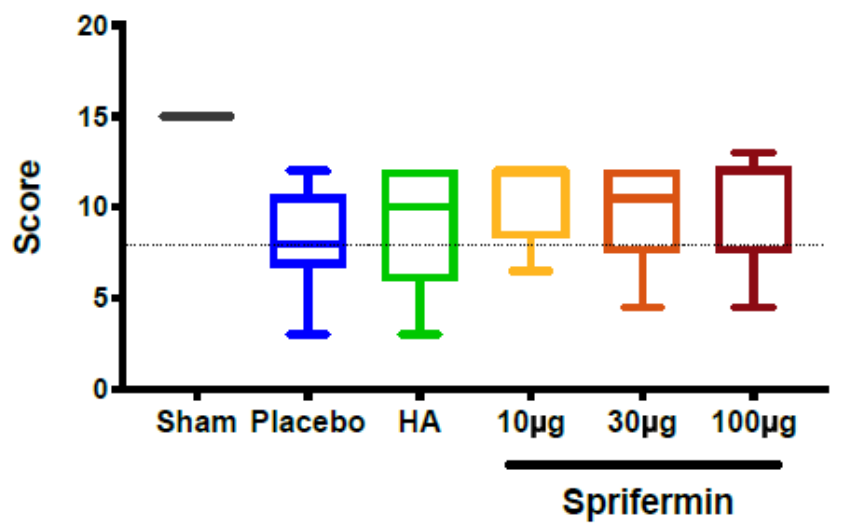

C

\section{Gross Morphology}

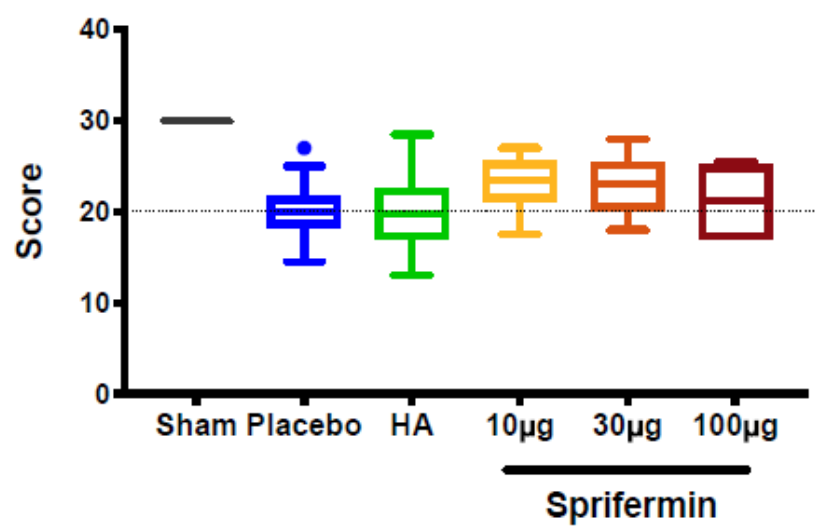

B

Arthroscopy

Combined Sprifermin

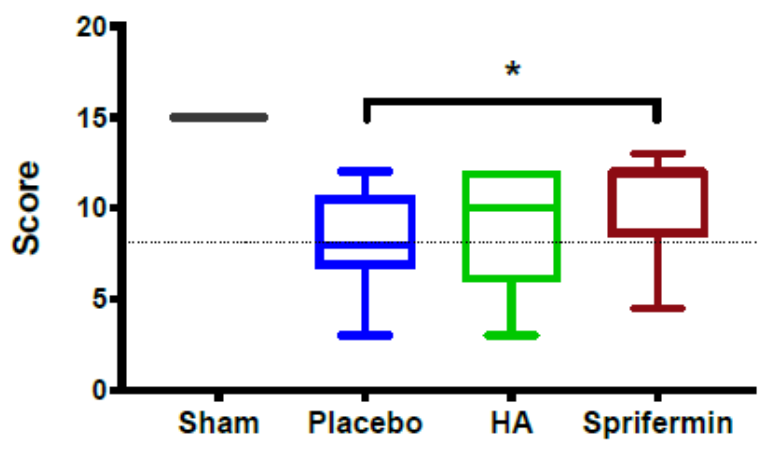

D

\section{Gross Morphology Combined Sprifermin}

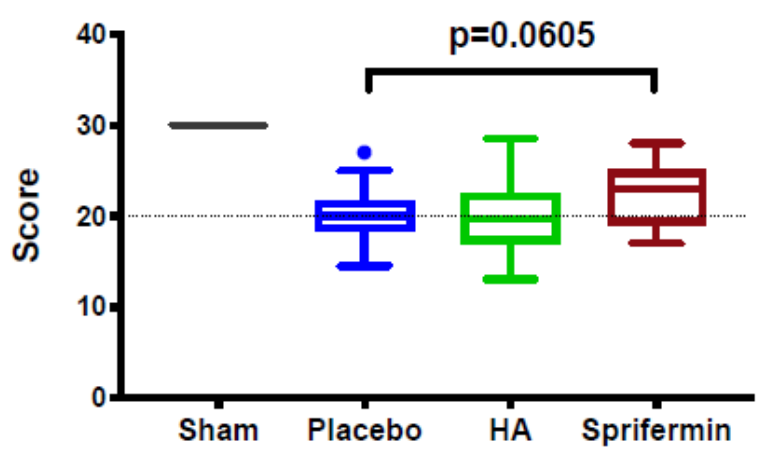

Figure 5

Second look Arthroscopic evaluation of the defect site at 12 weeks post-surgery and post-mortem gross evaluation (A) Sum-score of the three arthroscopic evaluation sub-scores showed no significant advantage in any single dose of sprifermin. (B) Arthroscopic evaluation sum-score of combined sprifermin groups was significantly higher than placebo. (C) Sum-scores of the six sub-scores showed no significant improvement in gross morphology following any of sprifermin doses. (D) Sum-scores showed a nearly significant improvement in gross morphology when scores from three sprifermin doses were combined. Dotted line indicates median of the placebo group. $n=6$ or $18,{ }^{\star} p<0.05$ by Kruskal-Wallis and Dunn's multiple comparison test. 
A

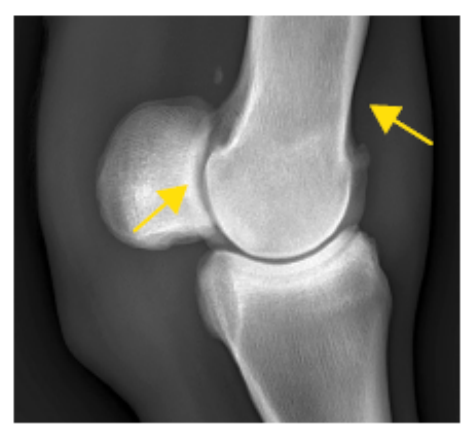

$\mathbf{F}$

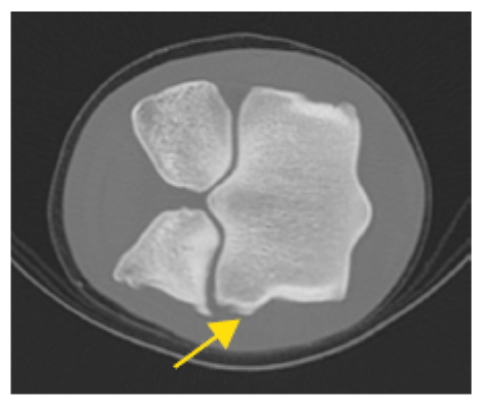

B

Radiography Week 12

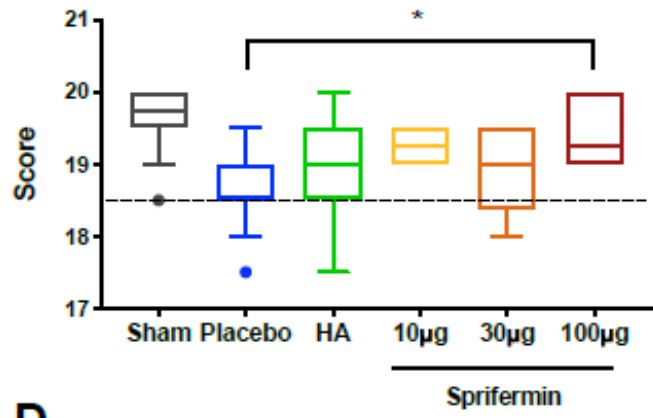

Radiography Week 24

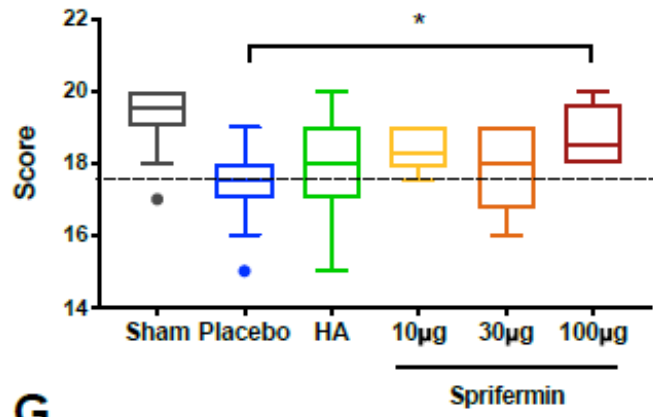

CT Scan

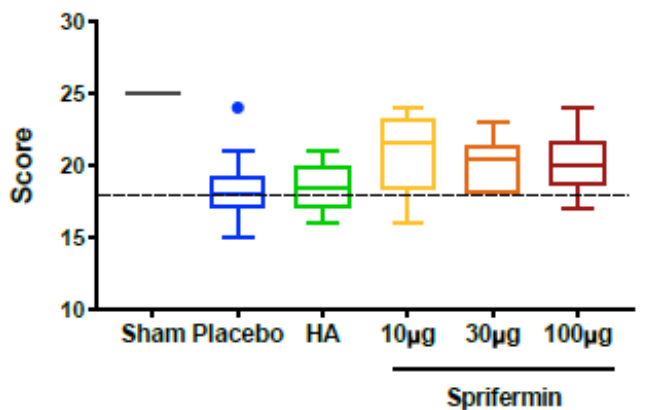

C

Radiography week 12

Combined Sprifrmin

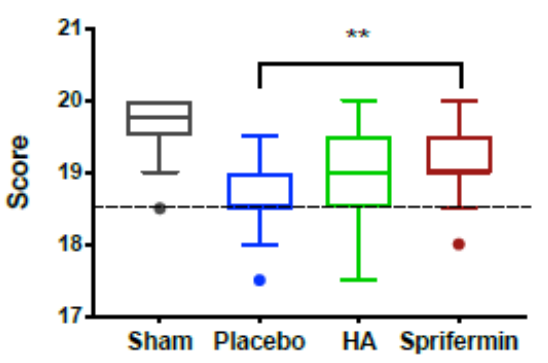

E

Radiography Week 24

Combined Sprifermin

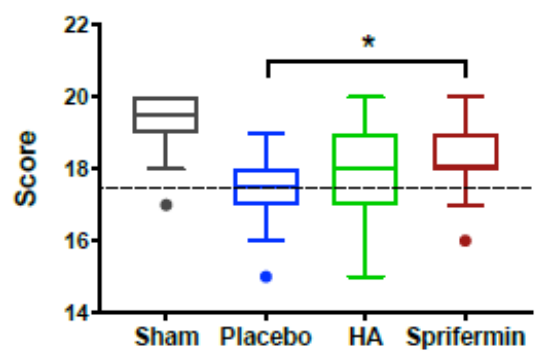

H

CT Scan

Combined Sprifermin

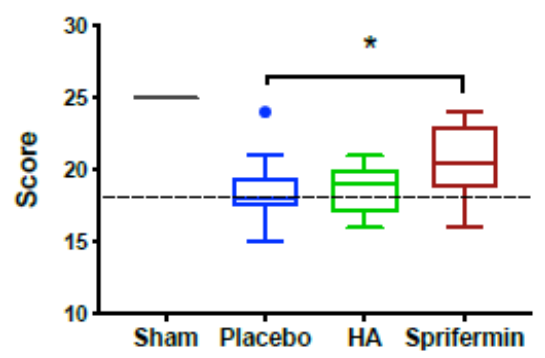

\section{Figure 6}

Radiographic and CT scan evaluation of fetlocks (A) Radiograph of a fetlock 24 weeks post-surgery showed formation of osteophytes (yellow arrows). (B) Evaluation of sum-scores of four radiography sub-scores showed a significant improvement in $100 \mu \mathrm{g}$ sprifermin treatment group at 12 weeks post-surgery. (C) Combined scores from sprifermin treatment groups at 12 weeks post-surgery showed a significant improvement in radiographic scores. (D) Evaluation of sum-scores of four radiography sub-scores showed a significant improvement in 100 $\mu \mathrm{g}$ sprifermin treatment group 24 weeks post-surgery when compared to placebo. (E) Combined scores from sprifermin treatment groups at week 24 showed a significant improvement in radiographic scores. (F) CT-Scan image of a fetlock showed formation of osteophytes (yellow arrow). (G) Evaluation of sum-score of four CT scan sub-scores did not detect a significant improvement following treatment with any of three doses of sprifermin. (H) Sum-score of combined sprifermin groups showed a significant improvement compared to 
placebo. Dotted line indicates median of the placebo group. $n=6$ or $18,{ }^{*} p<0.05$ by Kruskal-Wallis and Dunn's multiple comparison test.

\section{Supplementary Files}

This is a list of supplementary files associated with this preprint. Click to download.

- Additinalfile3TableS3.docx

- Additionalfile7FigS3.pdf

- Additionalfile8FigS4.pdf

- Additionalfile1TableS1.docx

- Additinalfile2Tables2.docx

- Additionalfile6FigS2.pdf

- Additionalfile10FigS6.pdf

- Additionalfile9FigS5.pdf

- Additionalfile5FigS1.pdf

- Additionalfile4Tables4.docx 